\section{JTI}

JOURNAL OF

TRAUMA AND INJURY

\title{
Usefulness of Shock Index to Predict Outcomes of Trauma Patient: A Retrospective Cohort Study
}

\author{
Myoung Jun Kim, M.D., Jung Yun Park, M.D., Mi Kyoung Kim, M.D., \\ Jae Gil Lee, M.D., Ph.D.
}

Department of Surgery, Yonsei University College of Medicine, Seoul, Korea

Received: August 23, 2018

Accepted: September 18, 2018

\section{Correspondence to}

Jae Gil Lee, M.D., Ph.D.

Department of Surgery, Yonsei University College of Medicine, 50-1 Yonsei-ro,

Seodaemun-gu, Seoul 03722, Korea

Tel: $+82-2-2228-2127$

Fax: $+82-2-313-8289$

E-mail: jakii@yuhs.ac
Purpose: We investigated how prehospital, emergency room (ER), and delta shock indices (SI) correlate with outcomes including mortality in patients with polytrauma.

Methods: We retrospectively reviewed the medical records of 1,275 patients who visited the emergency department from January 2015 to April 2018. A total of 628 patients were enrolled in the study. Patients were divided into survivor and non-survivor groups, and logistic regression analysis was used to investigate independent risk factors for death. Pearson coefficient analysis and chi-square test were used to examine the significant relationship between SI and clinical progression markers.

Results: Of 628 enrolled patients, 608 survived and 27 died. Multivariate logistic regression analysis reveals "age" ( $p<0.001$; OR, 1.068), "pre-hospital SI $>0.9$ " $(p<0.001$; OR, 11.629), and "delta SI $\geq 0.3$ " ( $p<0.001 ; \mathrm{OR}, 12.869)$ as independent risk factors for mortality. Prehospital and ER SIs showed a significant correlation with hospital and intensive care unit length of stay and transfusion amount. Higher prehospital and ER SIs $(>0.9)$ were associated with poor clinical progression.

Conclusions: SI and delta SI are significant predictors of mortality in patients with polytrauma. Moreover, both prehospital and ER SIs can be used as predictive markers of clinical progression in these patients.

Keywords: Shock index; Trauma; Mortality; Biomarkers

\section{INTRODUCTION}

Many aspects are important in the management of trauma patients, but the most important aspect is the detection and control of bleeding. Massive bleeding is the most 
significant determinant of prognosis in patients with trauma and the most common cause of death. In other words, in the so-called golden hour, control of bleeding is the most essential step that can improve the patient's prognosis and reduce the risk of mortality [1,2].

However, control of bleeding is not a simple approach. Definite external bleeding by trauma can be controlled by on-site compression. However, internal bleeding resulting from internal organ injury and pelvic bone or major proximal bone fracture is often not detected because of various factors, and this causes delays in management $[3,4]$. When patients with trauma miss the timing of hemorrhagic control, coagulopathy or systemic acidosis may develop. Moreover, this lethal triad leads to death.

Recently, the concept of shock index (SI) has regained attention in the area of polytrauma patient management. SI was first developed by Allgöwer and Burri [5] in 1967: this value is calculated as the heart rate (HR) divided by systolic blood pressure (SBP), and not considered independent of HR or SBP. Generally, SI $<0.7$ is considered normal, and SI $>0.9$ is considered abnormal [6-9]. Based on its simplicity and clarity, studies have been conducted on a marker that predicts bleeding in patients with polytrauma and reflects the acute volume loss of hemorrhagic shock [7]. However, few studies have investigated differences in outcomes according to SI at different time points or scale of SI change.

We investigated the correlation between the SI at the pre-hospital trauma scenario and patient outcomes including mortality and between the SI at the inhospital emergency room (ER) and patient outcomes. Moreover, we also investigated the gap between ER and pre-hospital SIs, so-called delta SI, on whether it has a correlation with outcomes in patients with trauma.

\section{METHODS}

\section{Patient enrollment and data collection}

Records of consecutive patients aged 18 years or older with traumatic injury who were treated between January 2015 and April 2017 in an urban setting in Seoul, South Korea, were retrospectively reviewed. This study was approved by the Institutional Review Board (IRB No.
4-2018-0579), and the requirement for informed consent was waived because of the retrospective nature of the study.

A total of 1,275 trauma patients were admitted to the Emergency Department (ED) during the study period, and 628 patients were enrolled (Fig. 1). These patients were divided into survivor and non-survivor groups within 30 days and investigated the risk factor that caused the patient's death.

Then, we compared the differences in patients' progress according to the prehospital, ER, and delta SIs. Patients were divided into three groups: group with prehospital SI $<0.7$, those with prehospital SI $>0.7$ but $<0.9$, and those with prehospital SI $\geq 0.9$ [10]. Then, progressions of each group were compared. The progression variables investigated here are "hospital length of stay (LOS)", "intensive care unit (ICU) LOS", "transfusion amount within 24 hours", and "duration of mechanical ventilation (MV)." Subsequently, patients were divided into three groups again according to ER SI the same way as the investigated variables mentioned above. Finally, the patients were divided into two groups based on delta SI 0.3 point, and differences in progression between the two groups were compared.

\section{Clinical variables}

Data collection included the following variables: sex, age, injury mechanism, vital signs at the prehospital scene and ER, abbreviated injury scale (AIS) score, injury severity score (ISS), revised trauma score (RTS), trauma and injury severity score (TRISS), arterial blood values $(\mathrm{pH}$, $\mathrm{PCO} 2, \mathrm{PO} 2$, hemoglobin, base excess [BE]), and serum lactate level. We analyzed amount of transfusion within 24 hours, hospital LOS, ICU LOS, duration of MV, and mortality within 30 days.

The prehospital SI was calculated by dividing the patient's HR by the SBP based on 119 medical records measured in the trauma scene. The ER SI was calculated by the same method as that in the prehospital SI based on the vital signs measured immediately after the ED visit. Finally, the delta SI was calculated as the "ER SI minus prehospital SI." 


\section{Statistical analysis}

Statistical analysis for investigated items was performed using SPSS ${ }^{\circledast}$ version 23.0 (IBM, Armonk, NY, USA). Categorical data were presented as numbers (\%) and compared using the chi-square or Fisher's exact tests. Continuous variables were expressed as mean \pm standard deviation or median and interquartile range, and the data were compared between groups using the Student $t$-test or Mann-Whitney $U$ test. Factors found to be significantly associated with mortality on univariate analysis were included in multivariate analysis. Logistic regression modeling was performed using the maximum likelihood method and backward stepwise selection. Goodness of fit was assessed using the Hosmer-Lemeshow test. The odds ratios (ORs) were given with 95\% confidence intervals (CIs). Kaplan-Meier survivor curves were generated using the mortality and hospital LOS to compare the survivor of each group, and the hazard of death was compared across the groups using the log-rank test. Pearson correlation coefficients were used to evaluate the relationship between SI and several outcome variables. A $p$-value $<0.05$ was considered statistically significant.

\section{RESULTS}

\section{General characteristics}

A total of 628 patients were included in this study. Among them, 453 were male and 175 were female. The mean ISS was 13.12 , the mean RTS was 7.356 , and the mean TRISS was 91.59. The median ICU LOS was 4 days, and the median hospital LOS was 7 days.

The baseline characteristics of patients are presented in Table 1. Of 628 patients, 27 patients died within 30 days, and 601 survived. Overall mortality was $4.49 \%$. There was no significant difference in sex $(p=0.076)$, but there
1275 patients (Trauma CP activated Pt.)
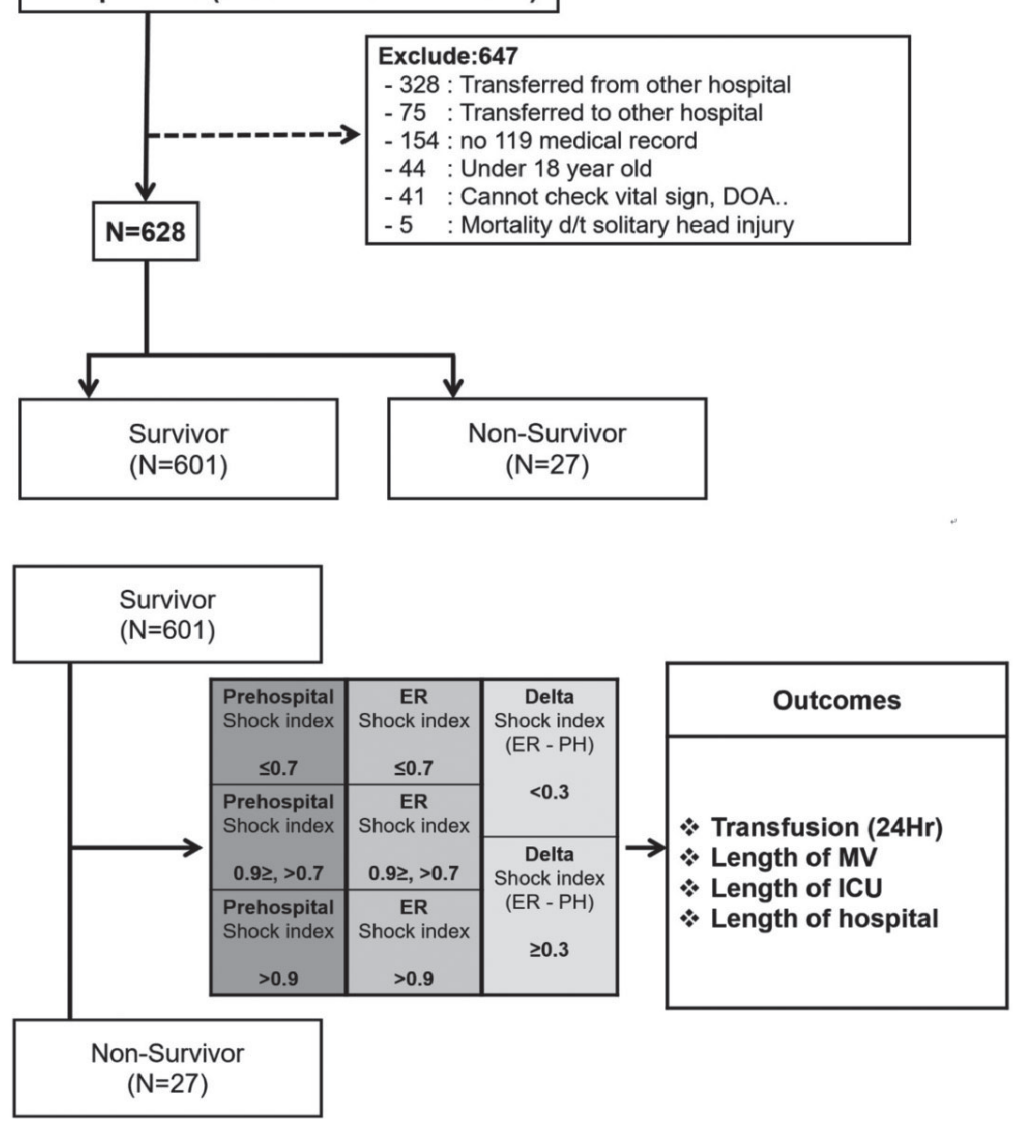

Fig. 1. Flow diagram of patients selected for analysis. CP: critical pathway, Pt:: patient, DOA: death on arrival, d/t: due to, ER: emergency room, PH: pre-hospital, Hr: hour, MV: mechanical ventilation, ICU: intensive care unit. 
was a significant difference in mortality according to age $(p<0.001)$. There was a significant difference in mortality according to injury mechanism $(p=0.016)$, and pedestrian traffic accident (51.9\%) was the most common in the non-survivor group. The injured site was significantly as- sociated with mortality with respect to the chest $(p<0.001)$ and extremity $(p=0.021)$. ISS $(p<0.001)$, RTS $(p<0.001)$, and TRISS $(p<0.001)$ also showed significant differences between the two groups. $\mathrm{BE}(p<0.001)$, lactate $(p<0.001)$, and $\mathrm{pH}(p<0.001)$ also showed significant differences be-

Table 1. Baseline characteristics of patients

\begin{tabular}{|c|c|c|c|}
\hline & Survivor $(n=601$ ) & Non-survivor $(n=27)$ & $p$-value \\
\hline Age (years) & $46.1 \pm 19.1$ & $60.0 \pm 18.8$ & $<0.001$ \\
\hline Gender & & & 0.076 \\
\hline Male & $438(72.9)$ & $15(55.6)$ & \\
\hline Female & $163(27.1)$ & $12(44.4)$ & \\
\hline Injury mechanism & & & 0.016 \\
\hline MVA (pedestrian) & $149(24.8)$ & $14(51.9)$ & \\
\hline MVA (motorcycle) & $135(22.5)$ & $0(0)$ & \\
\hline MVA (passenger) & $77(12.8)$ & $2(7.4)$ & \\
\hline Bicycle accidents & $26(4.3)$ & $0(0)$ & \\
\hline Falls & $146(24.3)$ & $8(29.6)$ & \\
\hline Stab (penetrating) & $35(5.8)$ & $2(7.4)$ & \\
\hline Others & $33(5.5)$ & $1(3.7)$ & \\
\hline \multicolumn{4}{|l|}{ AIS } \\
\hline Head and neck & $1.35 \pm 1.50$ & $2.15 \pm 2.03$ & 0.055 \\
\hline Face & $0.44 \pm 0.83$ & $0.81 \pm 1.18$ & 0.113 \\
\hline Chest & $0.95 \pm 1.41$ & $2.70 \pm 1.35$ & $<0.001$ \\
\hline Abdomen & $0.73 \pm 1.21$ & $1.07 \pm 1.39$ & 0.209 \\
\hline Extremities & $0.97 \pm 1.24$ & $1.81 \pm 1.78$ & 0.021 \\
\hline External & $0.83 \pm 0.48$ & $0.81 \pm 0.56$ & 0.864 \\
\hline ISS & $12.44 \pm 11.20$ & $28.15 \pm 14.01$ & $<0.001$ \\
\hline RTS & $7.493 \pm 0.907$ & $4.323 \pm 2.687$ & $<0.001$ \\
\hline TRISS (\%) & $93.87 \pm 14.66$ & $40.81 \pm 30.52$ & $<0.001$ \\
\hline \multicolumn{4}{|l|}{$A B G A$} \\
\hline $\mathrm{pH}$ & $7.370 \pm 0.085$ & $7.082 \pm 0.162$ & $<0.001$ \\
\hline $\mathrm{BE}$ & $-5.25 \pm 3.75$ & $-16.06 \pm 5.06$ & $<0.001$ \\
\hline Lactate & $2.81 \pm 2.04$ & $8.32 \pm 4.99$ & $<0.001$ \\
\hline PCO2 & $36.36 \pm 8.68$ & $55.95 \pm 21.18$ & $<0.001$ \\
\hline $\mathrm{PO} 2$ & $93.59 \pm 31.43$ & $63.84 \pm 36.73$ & $<0.001$ \\
\hline $\mathrm{Hb}$ & $13.35 \pm 2.24$ & $9.463 \pm 3.06$ & $<0.001$ \\
\hline SI (pre-hospital) & $0.72 \pm 0.25$ & $0.92 \pm 0.40$ & 0.020 \\
\hline SI (ER) & $0.73 \pm 0.27$ & $1.14 \pm 0.60$ & 0.002 \\
\hline Delta SI & $0.013 \pm 0.223$ & $0.2196 \pm 0.696$ & $<0.001$ \\
\hline
\end{tabular}

Values are presented as mean \pm standard deviation or number (\%).

MVA: motor vehicle accident, AIS: abbreviated injury scale, ISS: injury severity score, RTS: revised trauma score, TRISS: trauma and injury severity score, ABGA: arterial blood gas analysis, BE: base excess, Hb: hemoglobin, Sl: shock index, ER: emergency room. 
tween both groups. Prehospital ( $p=0.020)$, ER $(p=0.002)$, and delta SIs $(p<0.001)$ were all significantly higher in the non-survivor group.

\section{SI as a risk factor of inhospital mortality}

Mortality rate in patients with trauma was significantly higher when "prehospital SI was $>0.9(p<0.001)$ ", "ER SI $>0.9(p<0.001)$ ", and "delta SI $\geq 0.3(p<0.001)$ " (Table $2)$. Results of the univariate and multivariate regression analyses are shown in Table 3. Patient's age (OR, 1.068; 95\% CI, 1.038-1.099; $p<0.001$ ), "prehospital SI $>0.9$ " (OR,
11.629; 95\% CI, 4.195-32.235; $p<0.001)$, and "delta SI $\geq$ 0.3" (OR, 12.869; 95\% CI, 4.931-33.588; $p<0.001$ ) were identified as risk factors for trauma-related mortality. Kaplan-Meier curves showed significantly lower survival rate when prehospital SI was $>0.9(p<0.001)$, ER SI $>0.9$ $(p<0.001)$, and delta SI $\geq 0.3$ ( $p<0.001)$ (Fig. 2).

\section{SI and clinical progression}

Pearson coefficient analysis reveals significant linear relationship between prehospital SI and hospital LOS, ICU LOS, and transfusion amount within 24 hours (Table 4).

Table 2. Mortality according to SI

\begin{tabular}{|c|c|c|c|c|}
\hline & Survivor $(n=601)$ & Non-survivor $(n=27)$ & Mortality rate (\%) & $p$-value \\
\hline SI (pre-hospital) & & & & $<0.001$ \\
\hline$\leq 0.7$ & $352(58.6)$ & $10(37.0)$ & 2.84 & \\
\hline$\leq 0.9$ and $>0.7$ & $150(25.0)$ & $4(14.8)$ & 2.67 & \\
\hline$>0.9$ & $99(16.5)$ & $13(48.1)$ & 13.13 & \\
\hline SI (ER) & & & & $<0.001$ \\
\hline$\leq 0.7$ & $329(54.7)$ & $7(25.9)$ & 2.13 & \\
\hline$\leq 0.9$ and $>0.7$ & $161(26.8)$ & $6(22.2)$ & 3.73 & \\
\hline$>0.9$ & $111(18.5)$ & $14(51.9)$ & 12.61 & \\
\hline Delta SI & & & & $<0.001$ \\
\hline$<0.3$ & 554 (92.2) & $13(59.3)$ & 2.81 & \\
\hline$\geq 0.3$ & $47(7.8)$ & $11(40.7)$ & 18.97 & \\
\hline
\end{tabular}

Values are presented as number (\%).

SI: shock index, ER: emergency room.

Table 3. Risk factors of mortality in trauma patient

\begin{tabular}{|c|c|c|c|c|}
\hline \multirow{2}{*}{ Characteristic } & \multicolumn{2}{|c|}{ Univariate analysis } & \multicolumn{2}{|c|}{ Multivariate analysis } \\
\hline & OR $(95 \% \mathrm{Cl})$ & $p$-value & OR $(95 \% \mathrm{Cl})$ & $p$-value \\
\hline Age & 1.039 (1.017-1.062) & $<0.001$ & $1.068(1.038-1.099)$ & $<0.001$ \\
\hline \multicolumn{5}{|l|}{ SI (pre-hospital) } \\
\hline$\leq 0.7$ & Ref. & & Ref. & \\
\hline$\leq 0.9,>0.7$ & $0.936(0.290-3.040)$ & 0.916 & $2.045(0.578-7.235)$ & 0.267 \\
\hline$>0.9$ & $4.622(1.968-10.858)$ & $<0.001$ & $11.629(4.195-32.235)$ & $<0.001$ \\
\hline \multicolumn{5}{|l|}{ SI (ER) } \\
\hline$\leq 0.7$ & Ref. & & & \\
\hline$\leq 0.9,>0.7$ & $1.752(0.579-5.297)$ & 0.321 & & \\
\hline$>0.9$ & $5.928(2.333-15.061)$ & $<0.001$ & & \\
\hline Delta SI $\geq 0.3$ & 8.104 (3.557-18.462) & $<0.001$ & 12.869 (4.931-33.588) & $<0.001$ \\
\hline
\end{tabular}

OR: odd ratio, Cl: confidence interval, Sl: shock index, Ref:: reference, ER: emergency room. 
ER SI also shows significant linear relationship with these three variables. Duration of MV was not related to any kind of SI. Moreover, delta SI did not show any significant linear relationship with these clinical progression markers. Patients with "prehospital SI $>0.9$ " and "ER SI $>0.9$ " also showed significant difference in hospital LOS, ICU LOS, and transfusion amount in the analysis of Pearson chi- square test (Table 5).

\section{DISCUSSION}

Trauma is the number one cause of death in patients under 45 years of age. This is a common phenomenon in

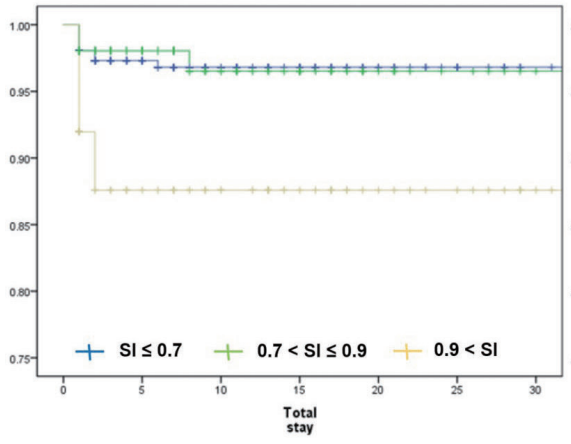

Prehospity SI

Log rank test: $p<0.001$

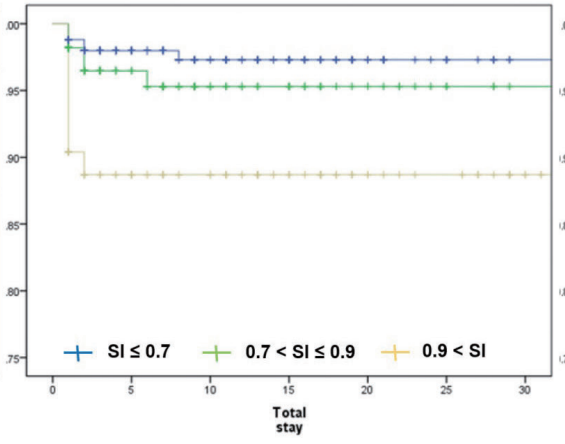

ER SI

Log rank test: $p<0.001$

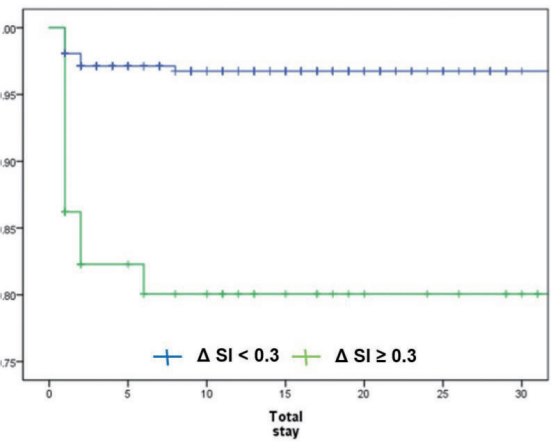

Delta SI

(ER SI - prehospital SI) Log rank test: $p<0.001$

Fig. 2. Kaplan-Meier survivor curve according to SI. Sl: shock index, ER: emergency room.

Table 4. Progression markers according to SI (Pearson coefficient analysis)

\begin{tabular}{|lcccc|}
\hline & Hospital LOS & ICU LOS & Duraion of M.V. & Transfusion (24 hour) \\
\hline SI (pre-hospital) & $0.201(p<0.001)$ & $0.212(p=0.001)$ & $0.084(p=0.358)$ & $0.351(p<0.001)$ \\
SI (ER) & $0.218(p<0.001)$ & $0.168(p=0.007)$ & $0.047(p=0.609)$ & $0.333(p<0.001)$ \\
Delta SI (ER-pre-hospital) & $0.052(p=0.191)$ & $-0.026(p=0.677)$ & $-0.033(p=0.718)$ & $0.011(p=0.902)$ \\
\hline
\end{tabular}

SI: shock index, LOS: length of stay, ICU: intensive care unit, M.V.: mechanical ventilation, ER: emergency room.

Table 5. Progression markers according to SI (Pearson chi-square test)

\begin{tabular}{|lcccc|}
\hline & Hospital LOS & ICU LOS & Duration of M.V. & Transfusion (24 hour) \\
\hline SI (pre-hospital) & $(p=0.003)$ & $(p=0.043)$ & $(p=0.735)$ & $(p=0.040)$ \\
$\leq 0.9(n=516)$ & $13.15 \pm 22.40$ & $7.54 \pm 9.45$ & $10.95 \pm 17.61$ & $4.05 \pm 4.37$ \\
$>0.9(n=112)$ & $22.73 \pm 32.27$ & $10.88 \pm 15.88$ & $12.13 \pm 18.46$ & $7.16 \pm 10.38$ \\
SI (ER) & $(p<0.001)$ & $(p=0.023)$ & $(p=0.191)$ & $(p=0.022)$ \\
$\leq 0.9(n=503)$ & $11.65 \pm 17.98$ & $7.09 \pm 9.34$ & $9.26 \pm 9.50$ & $3.84 \pm 3.33$ \\
$>0.9(n=125)$ & $27.74 \pm 39.57$ & $11.30 \pm 14.96$ & $13.84 \pm 24.20$ & $7.00 \pm 10.24$ \\
Delta SI (ER-pre-hospital) & $(p=0.093)$ & $(p=0.996)$ & $(p=0.950)$ & $(p=0.967)$ \\
$<0.3(n=570)$ & $14.00 \pm 22.33$ & $8.41 \pm 12.13$ & $11.39 \pm 14.70$ & $11.15 \pm 26.34$ \\
$\geq 0.3(n=58)$ & $23.24 \pm 40.65$ & $8.42 \pm 7.04$ & $5.33 \pm 7.73$ & $5.40 \pm 7.29$ \\
\hline
\end{tabular}

Sl: shock index, LOS: length of stay, ICU: intensive care unit, M.V.: mechanical ventilation, ER, emergency room. 
not only Korea but also the United States and the world [11-13]. Mortality rate reduction and improvement of outcomes depend on how quickly we detect and control bleeding in patients with trauma [14].

SBP and HR are sensitive indicators of bleeding or shock and can be easily measured in any situation. However, because of early physiologic compensation or pain in patients with trauma, it is difficult to determine the relationship between changes in these vital signs and actual bleeding $[3,4,7]$. Lactate and $\mathrm{BE}$ are also good indicators of patient's shock status; however, it takes about an hour to obtain results after arrival at the hospital $[15,16]$. Scoring system, such as ISS and TRISS, was also found to be highly correlated with the prognosis of patients with trauma; however, early diagnosis is limited because these scores are the final results after all workups have been completed [16,17]. An image study, such as computed tomography (CT), can show definite bleeding. However, $\mathrm{CT}$ is often difficult to perform depending on the patient's condition, and the advanced trauma life support guideline recommends not to perform $\mathrm{CT}$ at the primary surveillance stage [18].

The usefulness of SI was highlighted in the diagnosis of hemorrhage in patients with polytrauma for these reasons. Our study shows that SI is also useful in predicting clinical progress in patients with polytrauma.

SI was a good predictor of mortality in patients with polytrauma based on the results of our study. Especially, "delta SI" was identified as the most powerful predictor of trauma-related mortality. There were 58 (19\%) patients who had a delta SI of $\geq 0.3$, and their mortality rate was $18.97 \%$ in this study, which showed a significant difference from the group of patients with delta SI $<0.3(2.81 \%)$. Delta SI reflects the change in severity of trauma, and an increase in delta SI indicates hypovolemic shock, ongoing bleeding, and insufficient resuscitation during transfer $[11,19]$. Delta SI can be calculated only with vital signs monitoring without additional equipment, which is advantageous in the clinical decision in patients with acute stage trauma.

Patients who are elderly, taking $\beta$-blockers, or pregnant do not follow the usual physiology of bleeding and have different vital sign changes in a trauma situation. Because delta SI is calculated by differences measured at two points, its sensitivity is higher than that in the single cross-sectional SI when we evaluate patients with confusing factors. Moreover, patients with these confusing factors are generally at higher risk for mortality when trauma occurs. Therefore, delta SI is also useful in the survival of patients with high-risk trauma [19-21]. Moreover, delta SI has advantages of objective severity determination, triage, and sharing of patient severity information between the trauma scene and ED $[7,22]$. Thus, delta SI can play a role in predicting mortality and clinical decision-making in the early acute stage in patients with trauma-related hypovolemic shock.

However, delta SI did not show any significant difference in hospital LOS, ICU LOS, amount of transfusion, or duration of MV. We believe this is related to the trimodal peak of the mortality graph in patients with trauma. The second peak is death at the ED stage; a high delta SI is associated with second-stage mortality [23]. Therefore, delta SI is associated with acute stage outcome such as ED mortality rather than the long-term in-hospital progress.

However, SI at a single time point was significantly related to hospital LOS, ICU LOS, and transfusion amount. The higher the SI, the greater the difference in these outcomes of the patient: not only according to the ER SI but also along the prehospital SI. Unlike previous studies, this is meaningful in showing a significant correlation between prehospital SI and patient progress [14,21]. This result will help properly distribute the medical resources such as hemorrhagic control modality and blood transfusion to patients who most need them, among the patients with trauma transferred to the hospital [24]. Prospective study would be needed to determine the relationship between SI and various progression markers.

This is a retrospective study and has some limitations in the study of a single institution. In addition, the relatively small sample size and regional difference in trauma mechanism have to be considered as limitations of our results. Patients with trauma may have a different prognosis depending on the anatomical location of the bleeding, but in this study, this was not considered. Moreover, we did not investigate the use of $\beta$-blockers, which can affect the vital signs. Furthermore, when calculating the delta SI in this study, the time difference when patient was transferred from the trauma scene to the ED was not consid- 
ered. Thus, further study is needed to investigate how SI correlates with patient outcomes when the time variable is included.

However, our study is meaningful in that it was intended to show the difference in prognosis according to the different time points (prehospital and ER) of SI and change in SI (delta SI). This is a distinctive strength from the previous studies that attempt to correlate the outcome with a single measurement point of the SI. The results of this study on SI can help to predict the prognosis of patients with trauma earlier and provide treatment appropriately.

\section{CONCLUSION}

Prehospital, ER, and, especially, delta SIs can play a role as predictors of mortality in patients with trauma. Therefore, more active evaluation and management will be needed in patients with high SI.

Moreover, both prehospital and ER SIs can reflect the patient's hospital LOS, ICU LOS, and transfusion requirement. Thus, SI can be used to estimate the prognosis and provide appropriate treatment for each patient.

\section{ACKNOWLEDGEMENTS}

This study was supported by a faculty research grant of Yonsei University College of Medicine (6-2016-0100).

\section{REFERENCES}

1. Kauvar DS, Lefering R, Wade CE. Impact of hemorrhage on trauma outcome: an overview of epidemiology, clinical presentations, and therapeutic considerations. J Trauma 2006;60(6 Suppl):S3-11.

2. King RW, Plewa MC, Buderer NM, Knotts FB. Shock index as a marker for significant injury in trauma patients. Acad Emerg Med 1996;3:1041-5.

3. Victorino GP, Battistella FD, Wisner DH. Does tachycardia correlate with hypotension after trauma? J Am Coll Surg 2003;196: 679-84.

4. Rady MY, Smithline HA, Blake H, Nowak R, Rivers E. A com- parison of the shock index and conventional vital signs to identify acute, critical illness in the emergency department. Ann Emerg Med 1994;24:685-90.

5. Allgöwer M, Burri C. Shock index. Dtsch Med Wochenschr 1967;92:1947-50.

6. Rady MY, Nightingale P, Little RA, Edwards JD. Shock index: a re-evaluation in acute circulatory failure. Resuscitation 1992;23: 227-34.

7. Birkhahn RH, Gaeta TJ, Terry D, Bove JJ, Tloczkowski J. Shock index in diagnosing early acute hypovolemia. Am J Emerg Med 2005;23:323-6.

8. Rady MY, Rivers EP, Nowak RM. Resuscitation of the critically ill in the ED: responses of blood pressure, heart rate, shock index, central venous oxygen saturation, and lactate. Am J Emerg Med 1996;14:218-25.

9. Olaussen A, Blackburn T, Mitra B, Fitzgerald M. Review article: shock index for prediction of critical bleeding post trauma: a systematic review. Emerg Med Australas 2014;26:223-8.

10. Sasser SM, Hunt RC, Faul M, Sugerman D, Pearson WS, Dulski T, et al. Guidelines for field triage of injured patients: recommendations of the national expert panel on field triage, 2011. MMWR Recomm Rep 2012;61(RR-1):1-20.

11. Cannon CM, Braxton CC, Kling-Smith M, Mahnken JD, Carlton E, Moncure M. Utility of the shock index in predicting mortality in traumatically injured patients. J Trauma 2009;67:142630.

12. McNab A, Burns B, Bhullar I, Chesire D, Kerwin A. A prehospital shock index for trauma correlates with measures of hospital resource use and mortality. Surgery 2012;152:473-6.

13. Rhee P, Joseph B, Pandit V, Aziz H, Vercruysse G, Kulvatunyou N, et al. Increasing trauma deaths in the United States. Ann Surg 2014;260:13-21.

14. Singh A, Ali S, Agarwal A, Srivastava RN. Correlation of shock index and modified shock index with the outcome of adult trauma patients: a prospective study of 9860 patients. N Am J Med Sci 2014;6:450-2.

15. Kaplan LJ, Kellum JA. Initial pH, base deficit, lactate, anion gap, strong ion difference, and strong ion gap predict outcome from major vascular injury. Crit Care Med 2004;32:1120-4.

16. Manikis P, Jankowski S, Zhang H, Kahn RJ, Vincent JL. Correlation of serial blood lactate levels to organ failure and mortality after trauma. Am J Emerg Med 1995;13:619-22.

17. MacLeod J, Lynn M, McKenney MG, Jeroukhimov I, Cohn SM. Predictors of mortality in trauma patients. Am Surg 2004;70:805-10. 
18. ATLS Subcommittee; American College of Surgeons' Committee on Trauma; International ATLS working group. Advanced trauma life support $\left(\right.$ ATLS $\left.^{\circledR}\right)$ : the ninth edition. J Trauma Acute Care Surg 2013;74:1363-6.

19. Joseph B, Haider A, Ibraheem K, Kulvatunyou N, Tang A, Azim $A$, et al. Revitalizing vital signs: the role of delta shock index. Shock 2016;46(3 Suppl 1):50-4.

20. Pandit V, Rhee P, Hashmi A, Kulvatunyou N, Tang A, Khalil M, et al. Shock index predicts mortality in geriatric trauma patients: an analysis of the National Trauma Data Bank. J Trauma Acute Care Surg 2014;76:1111-5.

21. Zarzaur BL, Croce MA, Fischer PE, Magnotti LJ, Fabian TC.
New vitals after injury: shock index for the young and age $\mathrm{x}$ shock index for the old. J Surg Res 2008;147:229-36.

22. Mitra B, Fitzgerald M, Chan J. The utility of a shock index $\geq 1$ as an indication for pre-hospital oxygen carrier administration in major trauma. Injury 2014;45:61-5.

23. Cales RH, Trunkey DD. Preventable trauma deaths. A review of trauma care systems development. JAMA 1985;254:1059-63.

24. Vandromme MJ, Griffin RL, Kerby JD, McGwin G Jr, Rue LW 3rd, Weinberg JA. Identifying risk for massive transfusion in the relatively normotensive patient: utility of the prehospital shock index. J Trauma 2011;70:384-8; discussion 388-90. 